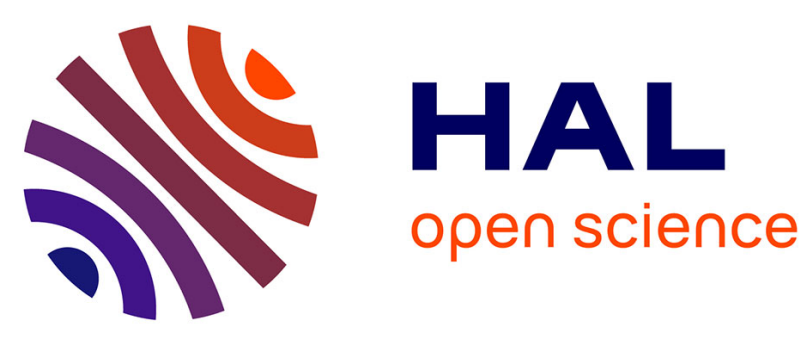

\title{
Les mobilisations de clavier
}

Romain Badouard

\section{To cite this version:}

Romain Badouard. Les mobilisations de clavier: Le lien hypertexte comme ressource des actions collectives en ligne. Réseaux: communication, technologie, société, 2013, 181, p.87-111. halshs01061962

\section{HAL Id: halshs-01061962 \\ https://shs.hal.science/halshs-01061962}

Submitted on 8 Sep 2014

HAL is a multi-disciplinary open access archive for the deposit and dissemination of scientific research documents, whether they are published or not. The documents may come from teaching and research institutions in France or abroad, or from public or private research centers.
L'archive ouverte pluridisciplinaire HAL, est destinée au dépôt et à la diffusion de documents scientifiques de niveau recherche, publiés ou non, émanant des établissements d'enseignement et de recherche français ou étrangers, des laboratoires publics ou privés. 


\title{
Les mobilisations de clavier
}

\section{Le lien hypertexte comme ressource des actions collectives en ligne}

\section{Draft Paper}

Ce document est la version intermédiaire d'un article dont la version finale a été publiée dans la revue Réseaux. Pour citer ou consulter la version finale : Badouard R., 2013, «Les mobilisations de clavier. Le lien hypertexte comme ressource des actions collectives en ligne », Réseaux, $\mathrm{n}^{\circ} 181, \mathrm{p} .87-111$.

http://www.cairn.info/resume.php?ID_ARTICLE=RES_181_0087

\author{
Romain Badouard \\ Centre de Sociologie de l'Innovation \\ Mines ParisTech \\ romain.badouard@mines-paristech.fr
}

\section{Résumé}

Les littératures académiques française et anglophone se sont intéressées à la question de l'action collective en ligne en accordant une attention particulière à l'évolution des formes de l'engagement, aux mutations des organisations militantes, ou encore à la production et la diffusion de contenus médiatiques alternatifs. La question de la structuration de publics en ligne, à travers l'étude des mécanismes facilitant la traduction d'un intérêt partagé pour une thématique, en une activité collective ayant pour but d'atteindre des objectifs spécifiques, semble avoir fait l'objet d'une attention plus disparate.

C'est de ce sujet que se propose de traiter cet article, en se focalisant sur une dimension bien précise des actions collectives sur internet : les usages stratégiques de liens hypertextes au sein des pratiques de mobilisation.

S'inspirant à la fois des apports de la littérature sur les pratiques politiques en ligne et des "link studies », cette étude repose sur un travail d'enquête s'appuyant sur un dispositif méthodologique qui articule étude statistique, analyse de contenu et « cartographie » du web. Elle démontre que les usages stratégiques du lien ont un effet structurant sur l'action et interviennent à la fois lors de la mobilisation d'un collectif (l'hypertexte comme " grappin » permettant de «drainer » des internautes vers une scène publique), de la conduite de l'action elle-même (l'hypertexte comme mécanisme agrégateur qui intègre des activités individuelles à un projet collectif), et de la constitution d'un espace public autour d'une scène (l'hypertexte comme canal de communication entre différents territoires thématiques). 


\section{Introduction}

Dans un article paru il y a près de 30 ans, Patrick Champagne livre son analyse d'une manifestation d'agriculteurs à Paris. Plutôt que de se focaliser sur son organisation ou son déroulement, le sociologue s'intéresse à la façon dont les organisateurs entendent transformer le cortège des manifestants en un véritable "événement politique », en obtenant des journalistes présents une couverture médiatique optimale. Il avait à l'époque proposé la notion de «manifestation de papier » pour rendre compte de la manière dont des collectifs militants intègrent des stratégies de médiatisation au sein de leurs répertoires d'action. Depuis, la question des transformations de ces répertoires d'action, au vu des bouleversements médiatiques et des évolutions technologiques, a constitué un prisme privilégié des travaux portant sur les mouvements sociaux et les actions collectives (Neveu, 1999).

La sociologie des usages et les études de communication se sont notamment saisies de la question en s'intéressant aux modalités de reconfiguration des activités militantes face à la généralisation des TIC. Différentes pistes de recherche ont fait l'objet d'une exploration poussée, comme l'évolution du rôle des organisations dans la conduite des actions collectives (Castells, 2002 ; Granjon, 2002a, Flanagin, Stohl, Bimber, 2006), les mutations des pratiques de mobilisation induites par l'apparition de nouveaux outils (Ollitrault, 1999; Granjon, $2002 \mathrm{~b}$; Boure et Bousquet, 2010) ou encore les formes alternatives de production médiatique (Jenkins, 2006 ; Allard et Blondeau, 2007 ; Cardon et Granjon, 2010).

Le sujet que nous souhaitons aborder dans cet article est transversal à ces différentes approches et a fait l'objet, dans les travaux précédemment cités, de questionnements croisés. Il s'agit de nous intéresser aux mécanismes de structuration des actions collectives en ligne, en nous focalisant sur les usages stratégiques des liens hypertextes. Notre clin d'œil à l'article de Champagne s'arrête donc au titre : nous ne traiterons pas ici de la charge symbolique des mobilisations sur internet, ni des productions médiatiques auxquelles elles donnent lieu, mais chercherons à saisir comment un intérêt partagé pour une thématique particulière se transforme en une activité collective ayant pour but d'atteindre des objectifs spécifiques. En nous focalisant sur les liens hypertextes, nous entendons décrire certaines dynamiques d'agrégation d'actions individuelles autour d'un projet collectif, afin de caractériser les modalités de constitution de publics « politiques » en ligne.

À cette fin, une piste de réflexion stimulante consiste à croiser les apports des travaux portant sur les actions collectives et ceux relevant des « link studies » (De Maeyer, 2011). Les « link studies » pourraient être définies comme l'ensemble des travaux qui s'intéressent aux liens hypertextes comme indicateurs ou révélateurs de dynamiques sociales et politiques sur le web. Ils ont notamment souligné la façon dont la répartition des liens équivalait à des formes de reconnaissance collective (Barabasi, 2002), ou conduisait à l'organisation de communautés autour d'affinités idéologiques (Fouetillou, 2008). Ils ont permis de mettre à jour une structuration spécifique de l'espace public en ligne, ou le lien hypertexte joue un rôle essentiel dans la mise en visibilité des informations : celui-ci constitue en effet autant un canal par lequel s'organisent des procédures d'évaluation de leur pertinence (Benkler, 2006), qu'une ressource pour les algorithmes des moteurs de recherche qui les hiérarchisent (Cardon, 2010). Ces phénomènes ont notamment été illustrés par les outils et méthodes de « cartographie » qui permettent de représenter une «écologie» du web, intégrant couches profondes, intermédiaires et visibles (Jacomy et Ghitalla, 2007 ; Ghitalla, 2008).

Nos précédents travaux ont quant à eux mis en évidence la dimension stratégique, et pas uniquement thématique, de l'organisation topologique du web. Celle-ci est en effet 
directement dépendante d'une distribution des liens hypertextes par des internautes, qui se livrent à des activités de «linking » selon des motifs très divers. C'est ainsi moins l'étude de la topologie du web qui permet de comprendre les formes d'action collective, que l'analyse de ces actions qui donne à voir un web en train de se structurer (Badouard et Monnoyer-Smith, 2013).

Nous cherchons dans cet article à analyser la façon dont les collectifs à l'origine de mobilisations ont utilisé des liens hypertextes pour sensibiliser des publics et structurer une action collective. Une telle étude se heurte à un verrou méthodologique : comment distinguer, sur le web, ce qui relève d'une action individuelle, d'une autre action individuelle articulée à une stratégie collective ? Après avoir répondu à cette question, nous observerons les formes de mobilisations et les différentes arènes dans lesquelles elles prennent forme, dans le cadre d'une consultation européenne (la Consultation Européenne des Citoyens) qui s'est déroulée en 2009 sur internet.

Ce cas d'étude s'ancre dans un contexte particulier. En 2006, suite aux non aux référendums sur le traité constitutionnel européen en France et aux Pays Bas, la Direction Générale Communication de la Commission entreprend la mise en œuvre d'un nouveau plan d'action, le Plan D comme Démocratie, Dialogue et Débat. Celui-ci vise notamment à organiser des consultations en ligne afin de réunir des citoyens « ordinaires » sur des plateformes web et à les faire débattre d'enjeux européens. Dans le cadre de la Consultation Européenne des Citoyens, il s'agissait plus précisément de mobiliser des internautes des 27 Etats membres afin des leur permettre de formuler des recommandations politiques à destination des décideurs européens, à travers une procédure délibérative reposant sur des forums et des outils de vote.

Au vu des objectifs de la Commission, la consultation s'est soldée par un double échec. Un échec d'audience tout d'abord, puisque pendant les 6 mois de la CEC, ce ne sont que 130000 visiteurs uniques qui se sont rendus sur la plateforme. Un échec de stratégie ensuite, puisque la Commission n'a pas réussi à toucher les citoyens européens " ordinaires » qu'elle visait avec cette expérience. Cette consultation a en effet été le théâtre de mobilisations de publics organisés qui ont déployé des stratégies de participation bien différentes de celles attendues par les organisateurs. Cette concurrence des stratégies laisse entrevoir une confrontation de différentes conceptions de l'espace public européen portées par les acteurs: une vision «agrégative» de la Commission qui consiste à additionner les opinions nationales pour donner corps à un "public européen», et une vision "segmentée» des militants qui s'organisent autour de sujets spécifiques au sein de réseaux thématiques hermétiques. Avant de revenir sur cette question, nous allons nous intéresser à ces mobilisations en montrant que les usages stratégiques du lien hypertexte jouent un triple rôle dans l'organisation et la conduite des actions collectives en ligne : le lien est à la fois un «grappin » qui sensibilise des internautes et les connecte au lieu de l'action, un mécanisme agrégateur qui articule des activités individuelles à un projet collectif, et un canal de communication entre différents réseaux de sites, structurant ainsi un espace public stratégique autour du site de la consultation. 


\section{Observer des actions collectives en ligne}

\section{Le « je » et le « nous»}

Le corrélat de toute action collective est le partage d'un objectif : c'est parce que des individus ont un intérêt commun, pouvant être modifié par la portée d'une action spécifique, qu'ils s'associent pour la conduire (Céfaï, 2007). En cela, les participants partagent un «sens du public ", c'est-à-dire une conscience de leur co-existence au sein d'une même entité (Céfaï et Pasquier, 2003). Afin d'influencer un état, ou une situation, en lien avec l'intérêt commun, ils s'engagent, se mobilisent, négocient, et partagent un ensemble de représentations à travers lesquelles ils construisent un collectif (Balme et Chabanet, 2002).

La principale spécificité des actions collectives en ligne a justement trait au fait qu'elles peuvent être le fruit d'individus n'ayant qu'une conscience très restreinte de leur appartenance à un collectif. Les formes de participation spécifiques au web, comme par exemple le relais d'informations sur les réseaux sociaux, ou l'approbation de messages par un clic, valorisent une implication individuelle aux dépens d'une diffusion de référentiels communs. L'action collective peut donc résulter d'actions non-coordonnées (Bimber, Flanagin \& Stohl, 2005), ou du moins se dérouler de façon opportuniste sans nécessiter un sentiment d'appartenance $a$ priori (Cardon, 2010). Nous pourrions ainsi parler de " collectifs malgré eux » pour exprimer l'idée qu'en ligne, le « sens du public » ne précède pas nécessairement la mobilisation.

Comment qualifier dès lors une «mobilisation de clavier»? Avant tout, celle-ci déborde le cadre d'activités militantes : elle concerne autant des citoyens « ordinaires » que des activistes organisés et présente des degrés de formalisation très variés. Elle consiste en une agrégation d'actions individuelles dans le cadre d'une activité dont l'objectif attendu est souhaité par l'ensemble des internautes qui y prennent part, dans la mesure où il répond à un intérêt commun, ou du moins à des intérêts partagés. Si l'idée d'une simple agrégation peut être considérée comme antinomique de cette idée de production d'un commun (Céfaï, 2007), il n'en demeure pas moins que sur le web, cette dimension est primordiale dans la mesure où les internautes sont seuls devant leur écran et mènent une action « au nom de ».

La «pratique individuelle» des actions collectives en ligne est d'ailleurs à mettre en perspective avec la notion d'engagement distancié proposée par Jacques Ion (1997), qui souligne le caractère flexible et peu contraignant des nouvelles pratiques de mobilisation. La structuration réticulaire d'internet, et les pratiques culturelles et citoyennes propres aux web, offrent un terrain particulièrement propice à l'expression de cet engagement distancié (Flichy, 2008 ; 2011). Le cas des pétitions en ligne (Ollitrault, 1999 ; Granjon, 2002b ; Boure et Bousquet, 2010 ; 2011), qui présente par ailleurs plusieurs similarités avec les pratiques que nous étudions dans cet article, en donne une bonne illustration. Voter une recommandation, comme signer une pétition, revient à mettre en visibilité un soutien à une position. La pratique de mobilisation consiste dans les deux cas à investir certains espaces du web (des blogs, des sites, des listes de diffusion, des réseaux sociaux), afin d'y mobiliser des internautes pour qu'ils se rendent sur les pages qui hébergent les recommandations ou les pétitions. Enfin, dans un cas comme dans l'autre, la dimension réellement collective de l'action est sujette à débat.

Pour Sylvie Ollitrault (1999) par exemple, les pratiques de pétition en ligne sont symptomatiques d'une déliquescence du «nous» militant et ne correspondent qu'à un engagement individuel. Fabien Granjon (2002b) relativise cet argument en soulignant que les 
pratiques de mobilisation en ligne correspondent justement à une stratégie de sensibilisation de publics non-militants, et que celles-ci n'ont qu'un impact limité sur les sociabilités militantes de terrain. Internet intègre un répertoire d'action composé de ressources hétérogènes, et sa spécificité réside dans sa capacité à mobiliser ponctuellement des individus au service d'un projet précis. Robert Boure et Franck Bousquet (2010) quant à eux insistent sur le fait que signer une pétition nécessite un engagement préalable quant à la thématique de la pétition, et que si l'action de signer est individuelle, la pétition en elle-même émane d'une communauté d'intérêt.

Si le débat sur le « je » ou le «nous» des actions collectives en ligne n'est pas tranché, un moyen de le contourner est de s'intéresser aux modalités de coordination entre ce « je » et ce « nous ». Dans leur analyse des collectifs militants émergeant sur le web dans le champ de la santé, Madeleine Akrich et Cécile Méadel (2007) distinguent trois types d'articulation d'individus à des collectifs. Le premier relève d'une reconnaissance collective de l'action : il en va ainsi, par exemple, lorsqu'un militant agit à titre individuel dans le but de faire reconnaître son acte par un mouvement. Le second se traduit par l'agrégation d'actions individuelles, lorsque des militants agissent isolément mais selon une stratégie collective. Le troisième correspond à une structuration en amont, quand les modalités et les formes de l'action sont décidées par un groupe constitué a priori. Rendre visible une action individuelle à un public constitue donc tout l'enjeu de l'action collective en ligne. Ce processus intègre trois étapes successives : le rassemblement d'individus autour d'intérêts communs, l'échange de messages entre ces individus et la coordination de leurs actions (Flanagin, Stohl \& Bimber, 2006).

En cela, le web fait évoluer les formes de l'action collective sur plusieurs aspects. En premier lieu, l'engagement est à la fois facilité et rendu flexible par l'atténuation des frontières entre sphères privée et publique : d'un côté, les contraintes matérielles de la mobilisation sont moindres (Castells, 2002) et de l'autre, la visibilité du soutien d'autres individus à une cause tend à favoriser l'implication personnelle (Margetts et al, 2009). Ensuite, le rôle des organisations s'en trouve limité, dans la mesure où les structures militantes ne sont plus forcément nécessaires à la conduite d'actions collectives (Bimber, Flanagin \& Stohl, 2005).

En revanche, en ligne comme hors ligne, le collectif se rend visible à lui-même sur une scène publique. Cette scène permet la construction d'une identité commune (Quéré, 1992), en favorisant la circulation de discours autant que d'images autour desquels se cristallisent des identités individuelles (Neveu, 1999).

Comme tout espace d'échange, leur dimension matérielle joue un rôle structurant dans la mesure où elle configure en partie les formats de prise de parole et les modalités d'action (Barry, 2001). En ligne, cette dimension est plus forte encore, puisque ces formats et ces modalités sont directement liés à des applications techniques qui les contraignent (Wright et Street, 2007), c'est-à-dire qui les rendent possibles tout en leur assignant des limites. Face à des sites politiques, cette configuration des actions et des interactions peut être assimilée à une "une incarnation matérielle des conceptions de la démocratie » (Monnoyer-Smith, 2010), dans la mesure où certaines représentations du citoyen y sont valorisées.

Ces scènes sont également ce qui permet, sur un plan méthodologique, à l'observateur d'appréhender comment des acteurs interagissent et se coordonnent (Céfaï, 2007). Elles constituent en cela des inscriptions matérielles des collectifs qui s'y sont formés (Brugidou,

\footnotetext{
${ }^{1}$ MONNOYER-SMITH, 2010, p.74.

${ }^{2}$ Cette analyse statistique a été rendue possible par l'accès à la plateforme Google Analytics du site de la
} 
2008 ; Benvegnu \& Brugidou, 2008). Les scènes publiques « en ligne » permettent de saisir a posteriori la structuration d'un public par le biais d'une description fine des formats d'action et d'interaction présents sur les sites au sein desquels ils agissent ou se réunissent. Cette posture méthodologique découle de la reconnaissance d'une forme de co-construction des actions militantes entre des ressources techniques et des motivations politiques. Par exemple, dans la continuité des travaux en sociologie des usages qui ont montré l'influence des dispositifs techniques dans l'engagement des internautes dans des régimes d'action (Jouët, 2011), Fabien Granjon (2002b) a souligné comment les technologies du web mettaient en œuvre des modes de coordination spécifiques qui donnaient forme aux collectifs constitués par leur biais. Dans notre cas d'étude, nous croisons ainsi cette description des modalités de coordination à une étude statistique des parcours de navigation ${ }^{2}$ et une analyse des contenus produits.

Notre terrain d'étude constitue dans ce cadre un cas particulièrement intéressant. Comme nous le disions en introduction, la Consultation Européenne des Citoyens (CEC) s'inscrit dans une stratégie de renouvellement des politiques de communication de la Commission qui, depuis le milieu des années 2000, visent à investir le web pour y mobiliser des citoyens « ordinaires» (Badouard, 2010). Par la mise en ligne de dispositifs participatifs, la Commission entend sensibiliser les internautes aux enjeux européens, et favoriser par le biais d'activités d'échange l'émergence d'un sentiment d'appartenance à une communauté transnationale, à même de combler un "déficit démocratique » qui affecte la légitimité des institutions. La CEC constitue à ce titre une «expérimentation démocratique » à part entière (Laurent, 2012), où il s'agit de donner corps à une opinion publique européenne via une procédure délibérative, et de mettre à l'épreuve un modèle de participation citoyenne au sein d'une plateforme en ligne (Badouard, 2013).

Or, le modèle participatif porté par la plateforme en ligne de la CEC est en contradiction avec la logique politique que nous venons de décrire : la plateforme européenne est déclinée en 28 sites nationaux ${ }^{3}$, cloisonnés les uns par rapport aux autres. Un internaute français par exemple ne peut pas échanger avec son homologue italien depuis son site national, et doit pour ce faire effectuer une double inscription. L'absence de canaux de circulation entre les différents sites bride donc l'émergence de dynamiques transnationales, et la contribution de la "société civile » européenne est conçue comme la somme des contributions des "sociétés civiles» nationales. A l'intérieur des espaces dédiés à chaque Etat membre, les internautes échangent au sein de forums, formulent des propositions qu'ils votent par la suite. Les 10 propositions les plus votées sont retenues pour des rencontres en face à face où des panels représentatifs des populations nationales rédigent à leur tour plusieurs propositions. Celles-ci sont ensuite mutualisées au niveau européen et de nouveau votées en ligne, avant d'être présentées par un panel de citoyens à un ensemble de décideurs européens (dont les présidents de la Commission et du Parlement), lors d'une rencontre à Bruxelles en mai 2009.

Tout l'enjeu de la phase en ligne est donc de produire dix recommandations plébiscitées par un nombre important d'internautes afin qu'elles constituent des éléments de débat pour les rencontres en face-à-face, d'où sortiront les recommandations finales. La procédure consultative en ligne, qui s'articule autour de trois étapes (l'information, le débat, le vote), conçue par l'agence La Netscouade, promeut une approche délibérative de la participation à

\footnotetext{
${ }^{2}$ Cette analyse statistique a été rendue possible par l'accès à la plateforme Google Analytics du site de la consultation, qui nous a été fourni dans le cadre d'une étude européenne sur la CEC, dont la partie en ligne était coordonnée par R. Kies (Université du Luxembourg) et S. Wojcik (Université Paris Est-Créteil).

${ }^{3}$ Un par Etat membre, mais deux pour la Belgique, l'un en Français et l'autre en Néerlandais.
} 
l'échelle des Etats membres, et agrégative à l'échelle européenne (Wojcik, 2011). Comme nous allons le voir, cette conception normative de l'espace public européen va être débordée par les pratiques de collectifs organisés.

\section{Quantifier une action collective}

Lors de notre enquête, nous avons réalisé un entretien avec les responsables de la phase en ligne de la consultation, deux jeunes salariés du média en ligne et partenaire du projet Toute l'Europe ${ }^{4}$. Pendant cet entretien, ceux-ci avaient exprimé leur désarroi quant à la mobilisation de groupes militants pour faire voter des propositions spécifiques. Ces pratiques avaient été perçues comme un véritable détournement du projet puisqu'elles étaient le fruit de collectifs organisés et non de citoyens "ordinaires", qui constituaient la cible première des organisateurs.

La plateforme de la CEC, en tant que scène publique, doit nous permettre d'appréhender les répertoires d'action mobilisés par ces collectifs (Tilly, 1986), c'est-à-dire les procédés qu'ils utilisent pour coordonner des actions et publiciser des informations sur cette scène. L'enjeu méthodologique est donc de réussir à quantifier ces actions, en distinguant notamment celles qui relèvent d'actions individuelles non-coordonnées, et celles qui relèvent d'une action collective. Pour ce faire, nous disposons d'un faisceau d'indices permettant d'appréhender l'impact de ces mobilisations sur le déroulement de la consultation. Prenons pour exemple le site français.

L'origine des visites constitue le premier indice dont nous disposons. D'après la plateforme Google Analytics, sur les 31901 visiteurs uniques ayant consulté le site français de la consultation entre le 1er janvier et le 31 mai $2009^{5}$, seulement $4,9 \%$ y sont arrivés par le biais de moteurs de recherche, contre $49,5 \%$ par des sites de référence, et $45,5 \%$ par accès direct. Un accès direct signifie en théorie que les internautes ont tapé (ou copié/collé) l'adresse du site dans leur navigateur afin de se rendre directement sur la page de la consultation ${ }^{6}$. En pratique, les moteurs qui comptabilisent et agrègent ces données recensent comme "accès direct» toutes les visites provenant d'une page qui n'a pas eu le temps de se charger complètement dans le navigateur. S'il est difficile de tirer des conclusions quant à ces « accès directs », on constate toutefois que la pratique dominante de navigation consiste à se diriger vers la consultation depuis d'autres sites, en suivant des liens hypertextes (et non à utiliser des moteurs de recherche).

Le recensement de ces sites d'origine peut être réalisé via un traçage des liens : en entrant l'adresse URL de la CEC sur un moteur de recherche, on obtient la liste de tous les sites qui proposent un lien vers le terrain observé ${ }^{7}$. Dans notre cas d'étude, la liste en question comprend 92 sites. L'intérêt est ici de les analyser afin de comprendre si ceux-ci sont plus à même de mobiliser des citoyens " ordinaires" ou des militants. Une telle analyse peut être conduite à travers l'étude de leur ligne éditoriale, le plus souvent présentée au sein des rubriques «Qui sommes-nous? » ou «A propos », présentes sur la quasi-totalité de ces sites ${ }^{8}$. Elle permet d'identifier les thématiques principales dont ceux-ci traitent, afin de les

\footnotetext{
${ }^{4}$ Cet entretien s'est déroulé avec S. Wojcik dans les locaux de Toute l'Europe, à Paris, le 13 mai 2009.

${ }^{5}$ Les données statistiques qui seront fournies par la suite concernent également cette période.

${ }^{6}$ L'utilisation de marque-pages est également comptabilisée en tant qu'accès direct.

${ }^{7}$ Pour avoir accès à la totalité des sites en question, il est nécessaire d'utiliser des moteurs spécialisés, comme par exemple Yahoo Site Explorer, qui permettent de comptabiliser les liens « inscrits » dans des bannières.

${ }^{8}$ Pour les sites qui ne disposaient pas d'une telle rubrique, nous avons étudié le contenu des dix derniers articles publiés.
} 
catégoriser en fonction. Nous avons ainsi recensé des sites «euro-centriques », qui traitent avant tout de l'actualité européenne, «citoyennistes», qui promeuvent des pratiques participatives, " thématiques », qui se focalisent sur des sujets spécifiques (ne touchant ni à l'Europe ni aux formes d'expression de la citoyenneté) et "généralistes », qui au contraire abordent une grande variété de sujets.

Il s'agit ensuite de repérer, à l'intérieur de ces sites, la façon dont le lien vers la consultation européenne est mis en contexte. Lorsque celui-ci est intégré au sein d'un article, il s'accompagne souvent d'une proposition d'action: on invite l'internaute à réaliser une certaine activité en cliquant sur ce lien. En ce sens, l'hypertexte implique un acte d' " interprétation actualisée dans un geste » ${ }^{9}$, pour reprendre l'expression d'Yves Jeanneret (2007), directement lié au texte qui accompagne le lien. Or, en analysant les articles, on constate que les différents sites valorisent différents types d'action : les sites euro-centriques, citoyennistes et généralistes, ont ainsi tendance à s'adresser à des citoyens ordinaires et à les inviter à débattre au sein des forums, afin d'éprouver leur citoyenneté dans un cadre européen, alors que les sites thématiques s'adressent à un collectif constitué et les poussent à voter des propositions en lien avec les sujets dont ils traitent. Autrement dit, les différents sites mobilisent différents publics, et mettent en place différentes stratégies quant à la consultation.

L'objectif est alors d'évaluer la proportion des publics thématiques qui viennent voter des propositions, par rapport aux publics généralistes, euro-centriques et citoyennistes, qui viennent débattre. La comparaison des activités de votes et de débats constitue donc un indicateur intéressant : plus les votes sont nombreux sur le site de la CEC, et plus la probabilité d'une mobilisation de publics thématiques sera importante. Or, sur le site français de la plateforme, les votes ont été 35 fois plus nombreux que les messages produits (40 087 votes pour 1163 messages). Cette tendance se vérifie dans la quasi-totalité des sites nationaux : sur l'ensemble des 28 sites de la plateforme CEC, les activités d'échange au sein des forums ont engendré la production de 5009 messages. Dans le même temps, les propositions ont occasionné 68585 votes $^{10}$. Il y a donc eu, sur l'ensemble des sites, près de 14 fois plus de votes que de messages.

Si le quotient nombre de débats/nombre de votes constitue un indicateur, il est nécessaire d'aller plus loin dans l'analyse en cherchant à appréhender la relation qui existe entre la mobilisation d'un public thématique et le nombre de votes reçus par la proposition qu'il soutient. Afin de comprendre cette relation, un nouveau traçage d'URL est effectué, à partir non pas de l'adresse de la page d'accueil du site de la consultation, mais de celles des propositions les plus populaires. Nous avons ainsi étudié un échantillon de 80 propositions, soit les 10 propositions les plus votées en Allemagne, en Autriche, en Bulgarie, en Espagne, en Finlande, en France, en Italie, et au Royaume-Uni.

Sur ces 80 propositions, une minorité (27) dispose de relais sur internet, c'est-à-dire de sites qui proposent un lien vers la page hébergeant la proposition. On constate donc que la majorité des propositions n'a pas été produite par des publics thématiques, qui auraient sollicité le relais de ces propositions sur d'autres sites. Pour autant, rapporté au nombre de votes reçus, il existe bien une corrélation entre les mobilisations et les propositions les plus populaires sur les huit sites cités.

\footnotetext{
${ }^{9}$ JEANNERET Y. (2007), Y a-t-il (vraiment) des technologies de l'information?, Presses du Septentrion, p.170.

${ }^{10}$ Nous souhaiterions remercier ici M. Karlsson, doctorant à l'université d'Örebro (Suède), avec qui nous avons collaboré dans le cadre d'un projet européen, et qui nous a permis d'utiliser ses propres relevés statistiques.
} 
Le graphique ci-dessous représente les sites d'origine de ces 80 propositions en fonction du nombre de votes que celles-ci ont reçu : 42 propositions ont obtenu moins de 100 votes, 22 entre 100 et 500, 10 entre 500 et 1000 et 6 en ont reçu plus de 1000 . Pour chacune de ces catégories est représentée la part des propositions qui bénéficient de relais provenant de sites classés comme «thématiques ».

\section{Part des propositions bénéficiant de relais sur des sites thématiques en fonction du nombre de votes reçus}

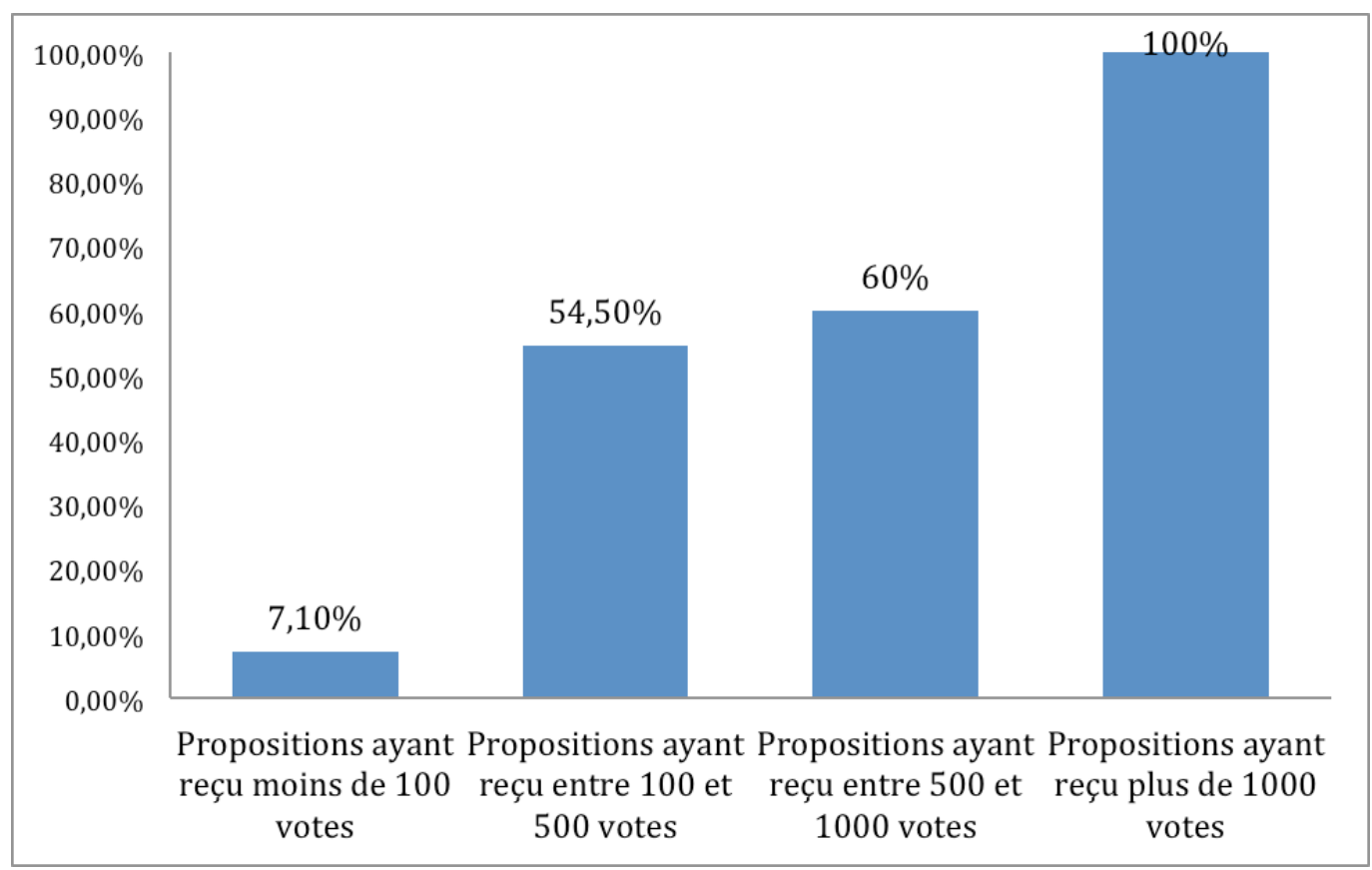

Parmi les propositions qui ont obtenu moins de 100 votes, seules 3 ont bénéficié de relais. La proportion augmente au fur et à mesure des votes reçus, jusqu'aux 6 propositions ayant obtenu plus de 1000 votes, qui bénéficient toutes de relais. Autrement dit, plus une proposition reçoit de votes, et plus forte est la probabilité qu'elle ait bénéficié de relais sur des sites thématiques. Ces propositions, si elles ne représentent que 1/3 des 80 étudiées, totalisent $77 \%$ des votes reçus. Les activités de votes sont donc avant tout produites par des publics thématiques qui circulent vers le site de la consultation via des liens hypertextes présents sur d'autres sites.

On peut en conclure que l'impact de la mobilisation de publics thématiques sur le déroulement de la consultation a été important car : (1) la plupart des visites sur la plateforme CEC ont pour origine d'autres sites (et non pas des moteurs de recherche); (2) ces sites invitent les internautes à réaliser différents types d'actions sur la plateforme CEC selon qu'ils soient euro-centriques, citoyennistes, généralistes ou thématiques ; (3) l'activité proposée par les sites thématiques (le vote), a été largement plébiscitée sur le site de la consultation, en comparaison des activités de débat valorisées par les autres sites ; (4) les propositions bénéficiant de relais sur des sites thématiques ont accaparé la grande majorité des votes, les qualifiant de fait pour la seconde phase de la consultation. L'intuition des salariés de Toute l'Europe est donc ici vérifiée de façon empirique : la stratégie de mobilisation de citoyens 
« ordinaires » portée par les responsables du projet est débordée par celle des publics thématiques.

\section{Mobiliser son public}

\section{Les pratiques militantes}

Si les actions de vote, reposant sur une mobilisation d'internautes via la distribution et l'agencement de liens hypertextes sur différents réseaux de sites, ont influencé la conduite de la consultation, il convient de décrire, dans une approche davantage qualitative, les formes que prennent ces mobilisations. Les pratiques de mobilisation ne sont pas uniquement le fait de collectifs militants organisés, elles peuvent également être mises en œuvre par des « amateurs », c'est-à-dire des internautes qui ne s'inscrivent pas dans une action coordonnée par une organisation. Dans les deux cas cependant, et comme dans le cas des pétitions en ligne que nous avons évoqué précédemment, il s'agit de conduire un ensemble d'internautes depuis un site spécifique vers la page d'une proposition afin que celle-ci recueille un maximum de votes. La diffusion des adresses URL des pages concernées se réalise via différents supports : listes de diffusion, réseaux sociaux, groupes en ligne, forums ou sites communautaires.

Commençons par les pratiques militantes, à travers l'exemple de la proposition arrivant en tête des votes sur le site français. Cette proposition, invitant à "Sortir du nucléaire et (à) favoriser les vraies énergies d'avenir $»^{11}$, a reçu 3829 votes $^{12}$. Là encore, le nombre de votes reçu apparaît pour le moins léger en comparaison des dizaines, voire des centaines de milliers de signatures que récoltent certaines pétitions en ligne ${ }^{13}$. Si dans le cas des pétitions hébergées sur des sites institutionnels, les taux de participation sont généralement plus faibles, il n'en demeure pas moins que les propositions les plus populaires reçoivent généralement plusieurs dizaines de milliers de signatures ${ }^{14}$. Pour autant, cet exemple nous permet d'analyser comment des organisations militantes diffusent des liens hypertextes afin de mobiliser des individus pour le vote d'une proposition.

La proposition en question a été postée sur le site par une certaine «Virgulea », dont le profil indique qu'elle est une femme de 30 ans vivant à Lyon et, surtout, qu'elle est membre du réseau militant «Sortir du nucléaire ${ }^{15}$. Le réseau en question est une organisation «parapluie » qui regroupe plus de 900 associations luttant contre le nucléaire. D'après la présentation du réseau sur son propre $\operatorname{site}^{16}$, ses principales modalités d'action relèvent de la production et de la diffusion d'informations, de la signature de pétitions, de l'organisation de manifestations, d'actions d'occupation de sites sensibles ou de blocage de convois de transport de déchets nucléaires. L'ensemble de ces actions relève d'une articulation entre du « en ligne » et du « hors ligne » : internet et les outils du web sont utilisés pour coordonner

\footnotetext{
${ }^{11}$ Accès : http://www.consultations-europeennes-des-citoyens.eu/fr/proposal/2433.

${ }^{12}$ Cette proposition est celle qui a reçu le plus de votes, tous sites nationaux confondus.

${ }^{13}$ Voir notamment les cas étudiés par Robert Boure et Franck Bousquet sur la pétition Vauzelle (2010) ou sur les pétitions contre le débat sur l'identité nationale (2011).

${ }^{14}$ Voir notamment les sites du 10 Downing Street au Royaume-Uni (http://epetitions.direct.gov.uk/petitions) ou de la Maison Blanche aux Etats-Unis (https://petitions.whitehouse.gov/petitions)

${ }^{15} \mathrm{~S}$ 'il est impossible de vérifier la véracité de ces informations, il est à noter que les participants n'étaient pas contraints de donner ce type d'information lors de leur inscription en ligne.

${ }^{16} \mathrm{http}: / /$ www.sortirdunucleaire.org/
} 
des manifestations «physiques », comme c'est souvent le cas dans le cadre des organisations militantes (Granjon, 2002b). Ici, nous allons nous focaliser sur une action menée uniquement en ligne.

La proposition du réseau a été postée le samedi 7 mars 2009 à 15 h58. Le samedi 7 et le dimanche 8, elle a été consultée 4 fois, puis 14 fois le lundi. Mais le mardi 10 mars, ce sont 4664 visites qu'a reçues la page qui l'hébergeait. Pour le mois de mars, la proposition antinucléaire est la plus consultée du site.

\section{Visites sur la page de l'ECC hébergeant la proposition «Sortir du nucléaire » entre le $1^{\text {er }}$ et le 31 mars 2009}

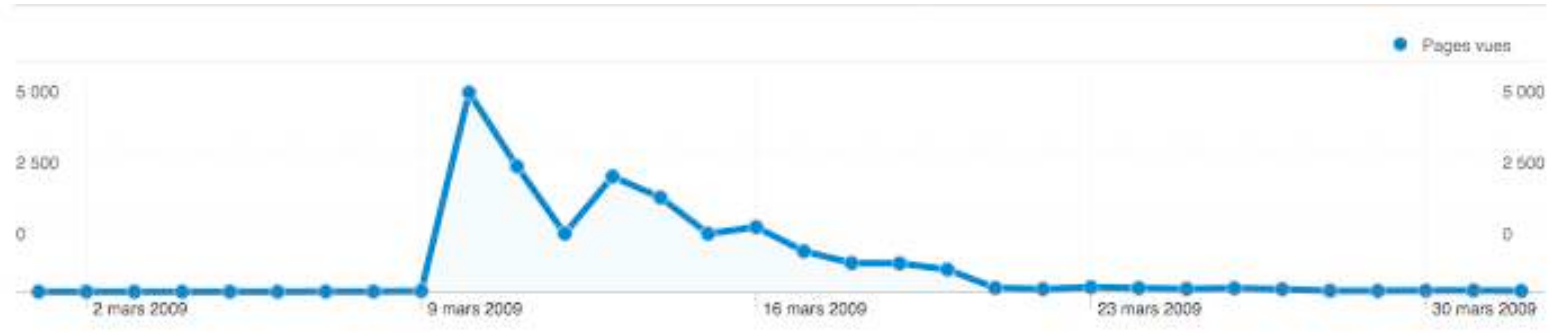

L'adresse URL de la page a été retrouvée sur 21 sites thématiques, pour la plupart des sites écologistes, certains appartenant à des organisations (les «Verts de Bretagne » ou «Bretagne Ecologie » par exemple), d'autres relevant de blogs personnels (mais traitant principalement de sujets liés à la protection de l'environnement). Lorsqu'on identifie les articles au sein desquels les liens ont été insérés, on s'aperçoit que l'ensemble de ces sites a en fait relayé un même communiqué de presse émanant du réseau «Sortir du nucléaire », rédigé par son directeur P. Brousse. La capture d'écran suivante représente ce communiqué de presse tel qu'il a été relayé sur le site «Brest-ouvert » le 10 mars 2009 : 


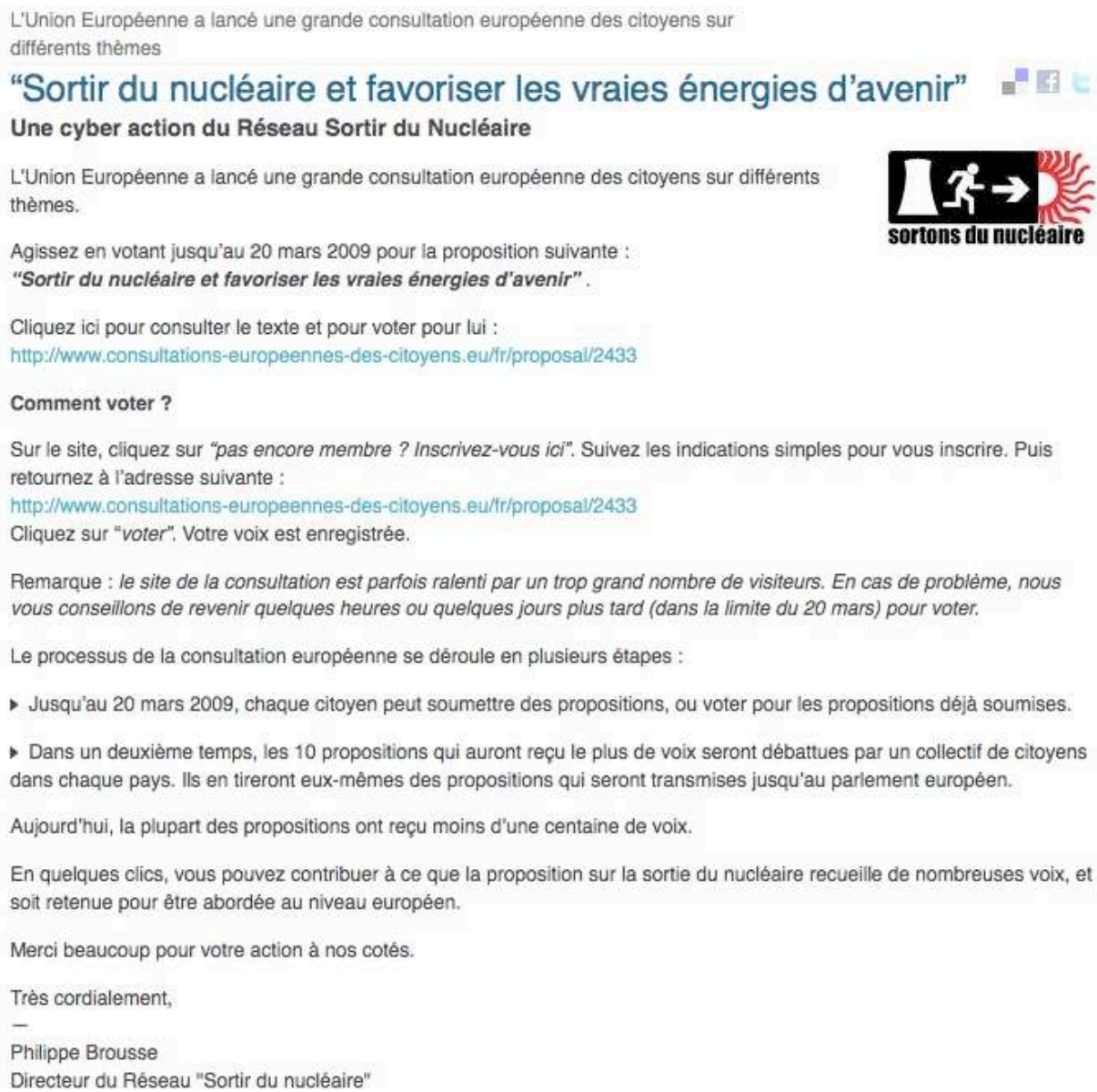

Parallèlement, les statistiques de la page hébergeant la proposition nous indiquent que, sur la période étudiée, $34,1 \%$ des visites ont eu pour origine une boîte courriel. De la même façon que les listes de diffusion sont utilisées dans le cadre de mobilisation d'individus pour voter des pétitions (Granjon, 2002b), on peut en déduire que ce même communiqué, ou un communiqué similaire, a été transmis par courriel à un ensemble d'individus inscrits sur la liste de diffusion de l'organisation, ou sur celles d'organisations « amies ».

Le scénario suivant se dessine : un membre du réseau « Sortir du nucléaire » rédige le 7 mars une proposition sur le site français de la consultation. Durant le weekend, le directeur diffuse dans son réseau et à des réseaux « amis » un communiqué de presse invitant leurs membres à se rendre sur le site et à voter la proposition en leur expliquant la démarche à suivre. Les réseaux amis relaient l'appel sur leur site. Par le biais des liens hypertextes ainsi diffusés, les membres s'inscrivent sur le site de la consultation et aboutissent à la proposition pour la voter. En quelques jours, elle devient la plus populaire et le restera jusqu'à la fin de la phase en ligne, ce qui la qualifie pour la seconde partie de la consultation.

Nous sommes ici face à une agrégation d'actions individuelles autour d'un projet collectif. Les internautes qui y sont engagés partagent un intérêt pour la question du nucléaire, et ont pour objectif commun la qualification de la proposition du réseau qu'ils soutiennent. La mobilisation, tout comme la conduite de l'action, reposent sur la diffusion de liens hypertextes qui doivent permettre aux internautes de se rendre sur le site de la consultation afin de voter la proposition. Cet usage stratégique du lien constitue un procédé intégrant un répertoire d'action en ligne. Cette action est structurée de façon «hiérarchique »: les 
dirigeants du réseau sont impliqués et la coordonnent d' «en haut 》 (via un communiqué de presse signé du directeur), en invitant les sympathisants à aller voter.

\section{Les pratiques amateurs}

D'autres pratiques, reposant sur le même procédé, relèvent de pratiques moins spécialisées, dans la mesure où elles sont le fait de militants ou sympathisants « de base ».

Prenons l'exemple de la $4^{\text {ème }}$ proposition la plus votée sur le site français, relative à la reconnaissance du «Droit de l'animal à ne pas être exploité ni tué ». L'adresse URL de la page hébergeant cette proposition a été retrouvée sur 10 sites thématiques, notamment sur un groupe Facebook concernant la «Défense des droits des animaux ». Les groupes Facebook sont structurés comme les pages personnelles, à partir d'un onglet « Mur» sur lequel les membres postent des messages et un onglet "Information» sur lequel les responsables présentent les raisons d'être du groupe. Mais ces groupes, à la différence des pages personnelles, disposent d'une troisième rubrique, intitulée « discussion », au sein de laquelle les membres peuvent créer et participer à des échanges de type «forum ». Une certaine Morgane G. y a créé un fil de discussion intitulé «Consultation européenne des citoyens : droits de l'animal» sur lequel elle a posté une liste d'adresses URL, menant à des propositions relatives à la défense des droits des animaux sur les sites de sept pays de la plateforme CEC. Elle introduit son message par le paragraphe suivant :

«Toujours dans le cadre de la consultation européenne des citoyens et, étant donné que, des 10 propositions arrivant en tête des suffrages dans chaque pays, 1 seule (la plus pertinente selon leur terme) se verra soumise à un vote au niveau européen (selon le choix d'un panel déterminé), j'ai donc pensé à optimiser les chances que la question du droit de l'animal ne passe pas à la trappe, en créant une proposition similaire dans plusieurs pays. »

S'en suit une liste de sept pays avec à chaque fois l'adresse à laquelle il faut se rendre pour s'inscrire, et l'adresse à laquelle il faut se rendre pour voter. La personne précise avoir écrit les cinq premières propositions et relayer deux autres propositions existantes, relatives à la protection des droits des animaux. Son message se termine par la phrase suivante: "Cette action ne prend que quelques instants, alors n'hésitez pas et votez! Et diffusion maximum demandée! ». Or, sur la page hébergeant la proposition française en question, les statistiques nous indiquent que $38,4 \%$ des visites ont effectivement été générées par Facebook. La mobilisation au sein de ce forum a donc eu un effet relativement important.

En comparaison du cas du réseau «Sortir du Nucléaire », la pratique de mobilisation est moins formalisée : on utilise des groupes présents sur les réseaux sociaux afin de toucher un public partageant un intérêt commun pour un sujet particulier, sans que celui-ci ne fasse pour autant preuve d'une appartenance à une organisation militante. L'action vient " d'en bas ", d'un simple membre, et on parie sur le relais de l'information via des réseaux personnels pour que l'action devienne réellement collective et prenne de l'ampleur. Ainsi, le recours aux réseaux sociaux semble à même de mobiliser un public plus «généraliste» que "thématique », dans la mesure où la circulation de l'information sur un site comme Facebook, par exemple, repose sur des affinités personnelles et non militantes. Lorsqu'on demande aux membres d'un groupe de relayer une information, ceux-ci vont poster cette information sur leur profil, rendant ainsi l'information visible à l'ensemble de leurs contacts. Or, ces «amis» ne sont pas (tous) des militants appartenant au groupe d'origine, et des personnes n'étant pas sensibilisées en premier lieu à la cause que défend le groupe vont être 
en contact avec l'information en question. Dans le cas présent, ces contacts vont se trouver invités à aller voter une proposition pour défendre les droits des animaux, alors qu'ils n'auraient peut-être jamais eu connaissance de cette action dans un autre contexte informationnel. Les formes de mobilisation spécifiques aux réseaux sociaux, où chaque membre fait appel à son cercle privé de contacts, qui feront à leur tour appel à leurs propres cercles, semblent favoriser un élargissement des publics, dans la mesure où la mobilisation se fonde non pas sur une affinité thématique/militante, mais sur une affinité personnelle.

Dans les deux cas que nous avons décrits, on observe que les publics sont en partie mobilisés dans des espaces privés et semi-publics : les militants ont recours à des listes de diffusion et donc à une communication qui relève de la sphère privée (à partir des boîtes courriels des inscrits), et les amateurs ont recours à des groupes Facebook qui, s'ils sont ouverts à tous, nécessitent une adhésion préalable, et surtout une inscription sur la plateforme de réseau social. En ce sens, ils constituent des espaces semi-publics, en « clair-obscur », pour reprendre l'expression de Dominique Cardon (2006).

Si les liens hypertextes constituent les canaux qui permettent de drainer les internautes depuis ces espaces vers la scène publique, tout l'enjeu est maintenant de déterminer la signification que les internautes attribuent à ces liens lorsqu'ils les cliquent. Comme nous le disions précédemment, cliquer un lien, c'est interpréter une proposition d'action. Dans le cas du Réseau Sortir du Nucléaire comme dans celui du message Facebook de Morgane G., l'association du lien à une incitation à l'action est claire : " Cliquez ici pour consulter le texte et pour voter pour lui » dans le premier cas, "cette action ne prend que quelques instants, alors n'hésitez pas et votez! » dans le second. L'internaute qui clique le lien en ayant lu le message se trouve alors dans deux situations : soit il adopte une démarche exploratoire, et suit le lien sinon par simple curiosité, du moins pour se représenter l'action qui lui est proposée, soit il le suit afin de réaliser l'action en question. Dans ce cas, il valide sa participation à l'action collective, en associant son action individuelle à un projet partagé par plusieurs internautes. Autrement dit, au-delà d'un canal de communication, le lien hypertexte est un mécanisme agrégateur qui permet l'intégration d'une volonté individuelle à une stratégie collective.

\section{Publics et territoires $d u$ web}

\section{L'européanisation des actions collectives}

Nous souhaiterions terminer cette analyse en observant la façon dont les liens hypertextes permettent de mettre en réseau différentes arènes et d'y faire circuler des publics. Dans le contexte européen de notre terrain, il est intéressant de réfléchir à la façon dont la forme spécifique que prennent les actions collectives sur le web peut, ou non, favoriser une « européanisation » des publics.

L'action collective à l'échelle européenne présente en elle-même des spécificités et les processus de «transnationalisation » sur lesquels elle repose englobent une grande diversité de dynamiques (Balme et Chabanet, 2002). Celles-ci peuvent en effet relever d'une coordination européenne d'acteurs qui protestent dans un cadre national, d'une coalition transnationale qui prend directement pour cible l'Union, ou encore de protestations intérieures contre des mesures communautaires (Imig \& Tarrow, 2002). Autrement dit, le caractère européen de l'action réside soit dans l'échelle de la mobilisation, soit dans la nature de 
l'institution dont on attend quelque chose ou contre laquelle on proteste. Dans tous les cas cependant, l'action garde une dimension nationale : si un ou deux composants de l'équation mobilisation/institution/politique publique sont européens, le ou les composants restants demeurent nationaux.

L'originalité des dynamiques que nous avons observées en ligne réside précisément dans cette déconnection entre les publics, les actions et les cadres géographiques qui habituellement les structurent. Ici, nous sommes aussi bien face à des mobilisations de publics nationaux pour mener des actions transnationales qu'à des mobilisations de publics internationaux pour mener des actions nationales.

Prenons quelques exemples pour éclaircir le propos. Premier cas : des internautes se rendent sur des sites, des groupes en ligne, ou des forums, qui sont fréquentés par des acteurs d'origines diverses et où l'échange se fait en plusieurs langues, mais qui portent sur une thématique bien spécifique. Sur ce site, l'internaute poste un lien vers une proposition sur un site national de la consultation afin d'engager les membres internationaux de la communauté à aller la voter. Par exemple, L'URL de la proposition ayant reçu le plus de votes sur le site espagnol, intitulée «Legalizacion de las drogas blandas ${ }^{17}$, a été retrouvée sur le forum du groupe «Welcome to Marihuna ${ }^{18}$ de la plateforme d'écoute musicale Last FM. Au sein de ce forum, les participants échangent majoritairement en anglais, mais aussi en espagnol, en allemand, en arabe ou en polonais. Tous partagent un intérêt (la consommation de cannabis) et sont enclins à s'engager dans une action pour un objectif commun (sa légalisation). L'individu ayant posté l'adresse de la proposition invitait (en espagnol) les membres du groupe à se rendre sur le site espagnol pour voter la proposition, indépendamment de leur nationalité.

Autre cas : la mobilisation d'un réseau (majoritairement) national afin de mener une action à une échelle transnationale. C'est le cas notamment du message de Morgane G. dont nous avons parlé : au sein d'un groupe Facebook, en français, elle invite les membres à aller voter des propositions relatives aux droits des animaux sur différents sites nationaux. Précisons que tous les sites nationaux sont configurés de la même façon et présentent les mêmes codes de navigation : il est donc facile de s'inscrire sur un site dont on ne maîtrise pas la langue, à partir du moment où l'on s'est inscrit préalablement sur son site national. Ici, la communauté française du groupe en question va mener une action européenne puisque l'objectif est de faire qualifier pour la seconde phase une proposition relative à la défense des droits des animaux dans d'autres pays.

Enfin, nous avons également observé un troisième cas, qui relève de la coordination d'acteurs nationaux pour mener une action transnationale. Ici, les internautes se rendent sur des sites d'envergure internationale et proposent aux internautes d'aller voter sur différents sites de la $\mathrm{CEC}$, indépendamment de leur nationalité. Ce type d'action peut également être relatif au relais d'un message à portée européenne dans des sphères nationales. Par exemple, l'URL de la seconde proposition la plus populaire sur le site français, " Abolir les corridas en Europe », a ainsi pu être retrouvée sur des sites militants à vocation internationale, comme le site de l'ALF, l'Animal Liberation Front. On la retrouve également sur un groupe Facebook espagnol intitulé « No mas crueldad en la corrida de toros que desparesca este horror »" ${ }^{19}$, et sur un forum germanophone. La proposition a été postée par «L'alliance anti-corrida»,

\footnotetext{
17 « Légalisation des drogues douces », trad. pers.

18 « Bienvenue à la Marijuana », trad. pers.

${ }^{19}$ « Mettre fin à la cruauté de la corrida afin que disparaisse cette horreur », trad. pers.
} 
réseau militant présent dans différents pays. D'après sa fondatrice, le réseau diffuse une newsletter comprenant près de 20000 inscrits présents notamment dans les 27 États membres de l'Union. Dans ce cas précis, la fondatrice du mouvement, ou un membre du réseau, a pris l'initiative de créer une proposition anti-corrida sur le site français, puis a invité, par le biais de sa newsletter, les sympathisants du réseau à voter la proposition ou à en créer des répliques. Les sympathisants en question ont relayé le message dans leur pays d'appartenance, produisant ainsi une ramification de l'action collective qui a suscité son européanisation.

Une variante de ce troisième type a trait aux actions structurées en amont directement à une échelle transnationale, comme cela a été le cas des réseaux liés à la promotion de l'esperanto. Sur les 28 sites nationaux de la $\mathrm{CEC}$, une proposition relative à l'esperanto se hisse aux premières places dans 22 cas. Elle est même la plus plébiscitée sur 15 de ces 22 sites. Une fois de plus, on retrouve les adresses relatives à ces propositions sur des sites et blogs en ligne. On les retrouve surtout au sein de groupes Yahoo et Google créés autour du thème de l'esperanto. Dans un des ces groupes, on peut notamment observer un fil de discussion se déroulant entre le 21 et le 28 janvier 2009. Les participants s'exprimant en esperanto, il ne nous a pas été possible de comprendre la teneur de ces échanges, mais ce que nous pouvons assurer, c'est que sont listés sur ces messages des liens vers des propositions relatives à l'esperanto sur 14 sites de la CEC. L'action collective semble avoir été structurée en amont: des individus de diverses nationalités postent sur des sites nationaux des propositions relatives à la promotion de l'esperanto puis partagent les liens vers ces propositions au sein de groupes en ligne constitués autour de cette même thématique. L'action semble donc dès le début structurée à une échelle transnationale, et les militants pro-esperanto se seraient coordonnés via des groupes en ligne, indépendamment de leurs nationalités.

On constate ainsi que l'usage stratégique de liens hypertextes comme ressources de mobilisation a pour conséquence de remodeler les formes relationnelles de l'action collective, à la fois en proposant de nouvelles modalités d'articulation entre activités individuelles et projet collectif, mais également en touchant des publics indépendamment de leur origine géographique. Contrairement aux actions européennes hors ligne qui restent liées à des territoires nationaux, la contrainte géographique se trouve ici sinon évacuée, du moins largement diminuée. Précisons ici que cet affranchissement est moins le produit d'un usage des liens qu'une particularité du web, et que cette déconnexion entre activisme et zones géographiques a été constatée dès les premières études sur les enjeux politiques d'internet (Castells, 1998). Ce qui est intéressant dans notre cas d'étude, c'est que l'articulation entre l'activité individuelle et le projet collectif engendrée par le lien rend possible une « coordination non coordonnée » d'individus (Bimber, Flanagin \& Stohl, 2005) quelque soit leur emplacement, qui de ce fait favorise l'européanisation des actions collectives.

Pour autant, la notion de territoire reste pertinente pour penser ces mobilisations, à partir du moment où on les comprend en tant que territoires thématiques, et non géographiques. Les publics se réunissent dans des lieux, des arènes en ligne (des groupes, des réseaux sociaux, des sites, des listes de diffusion) en fonction de centres d'intérêt qu'ils partagent, et c'est à partir de ces arènes qu'ils se mobilisent pour conduire une action sur une scène publique. 


\section{Espaces et réseaux}

Grâce à la « cartographie » du web, on peut représenter graphiquement des réseaux de sites inscrits dans des territoires thématiques ${ }^{20}$. Dans notre cas d'étude, nous avons sélectionné les 92 sites qui proposent des liens vers la consultation française, puis nous les avons " crawlés », c'est-à-dire que nous avons recensé les liens qu'ils proposaient vers d'autres sites ${ }^{21}$. La méthode de constitution de ce graphe est donc spécifique : on ne part pas de la consultation pour voir vers quels sites celle-ci pointe, mais on part des sites qui pointent vers la consultation et on observe vers quels autres sites ils pointent également. La carte suivante représente ainsi les territoires thématiques liés au site français de la consultation, dans la mesure où un ou plusieurs sites qui les composent y sont liés. Dans un souci de visibilité, nous avons enlevé du graphe le site de la CEC lui-même pour observer plus précisément les liens entre les différents réseaux.

\section{Cartographie des territoires thématiques liés à la Consultation Européenne des Citoyens}

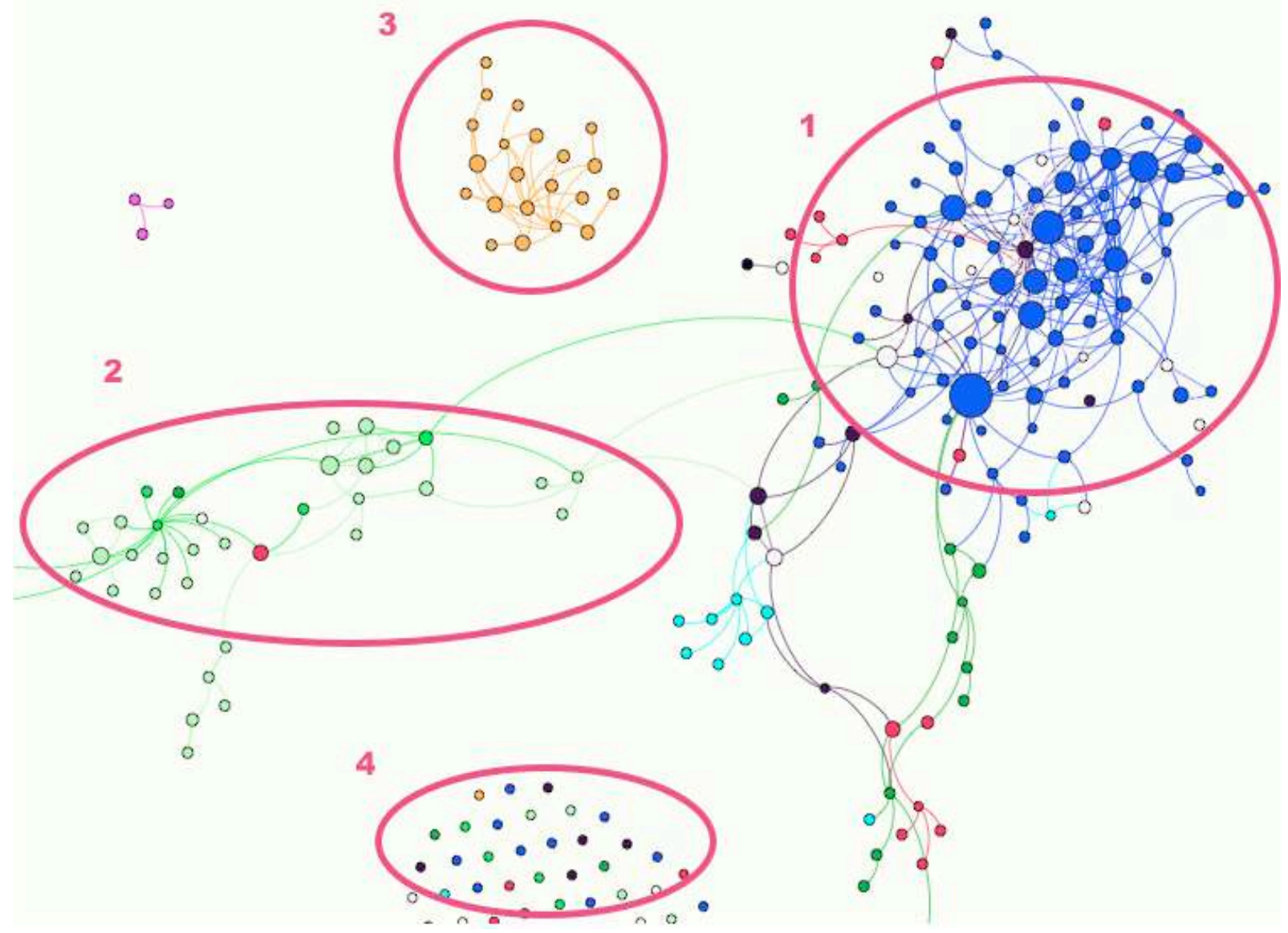

Le cercle $n^{\circ} 1$ représente les sites euro-centriques, c'est-à-dire l'ensemble de ceux qui abordent principalement des sujets européens. Ce réseau est dense (les différents sites sont très liés entre eux) et composé d'éléments hétérogènes en termes de types de site (on y retrouve aussi bien des médias spécialisés, que des sites institutionnels ou des blogs personnels). Les publics qui y circulent vont potentiellement être sensibilisés aux questions

\footnotetext{
${ }^{20}$ Nous souhaiterions remercier ici F. Pfaender qui nous a initié aux méthodes de « cartographie » du web.

${ }^{21}$ L'outil utilisé pour recenser les liens est le Navicrawler, développé par l'association WebAtlas : http://webatlas.fr/wp/navicrawler/
} 
européennes, et avoir tendance à se rendre sur le site français de la CEC afin de débattre (dans la mesure où ils sont susceptibles de parvenir sur le site de la CEC par le biais de sites eurocentriques).

Le cercle $\mathrm{n}^{\circ} 2$ représente le réseau écologiste, plus disparate et moins dense que le cercle eurocentrique (la couleur claire indique les réseaux de défense des droits des animaux et la couleur foncée les sites pouvant être assimilés à la mouvance écologiste). On observe que les différentes grappes, qui correspondent à différents courants, sont reliées non pas entre elles, mais au cercle euro-centrique via quelques points d'entrée. Le territoire thématique est donc ici faiblement structuré, mais il entretient des liens avec des sites institutionnels ou médiatiques traitant d'Europe, le plus souvent via des blogs de députés européens écologistes ou d'organisations politisées concernées par les politiques européennes en matière d'environnement. Ces blogs occupent ainsi un rôle de médiateur entre des publics thématiques et le cercle euro-centrique.

Le cercle $\mathrm{n}^{\circ} 3$ désigne des réseaux militant en faveur de la légalisation du cannabis (en orange), qui à l'inverse des réseaux écologistes sont isolés et n'entretiennent pas de liens avec le cercle euro-centrique. Le $n^{\circ} 4$ englobe un nombre important de sites pointant vers la CEC mais non reliés aux autres réseaux observés.

La «cartographie» du web ne permet d'illustrer qu'un nombre limité de parcours de navigation dans la mesure où ni la circulation d'internautes via des boîtes courriels ni l'usage des moteurs de recherche n'y sont représentés. Pour autant, elle permet de mettre à jour la variété des liens qu'entretiennent les différents territoires thématiques. Ceux-ci peuvent être isolés les uns des autres, ou au contraire permettre une circulation des internautes via des sites médiateurs. Mais ces derniers restent peu nombreux et notre représentation graphique tend à montrer que les différents publics évoluent dans des sphères séparées et ne se rencontrent qu'une fois arrivés sur le site de la consultation. Surtout, on constate que la façon dont ces réseaux s'articulent à la plateforme est directement liée à la stratégie des publics et aux actions qu'ils entendent y mener (ce sont les hypertextes menant vers des propositions spécifiques qui créent le lien entre les réseaux et la plateforme consultative).

Cette méthode spécifique de structuration de l'espace public de la consultation, ici compris comme l'ensemble des territoires thématiques liés à la plateforme qui l'organise, correspond à un procédé que nous avons baptisé "principe d'abordage»: des réseaux thématiques où s'activent des publics (les «abordants»), proposent via des liens hypertextes (les grappins) des canaux de circulation vers le site participatif (les « abordés ») pour y conduire une action de vote de propositions. Via les abordages successifs de différents réseaux se construit autour de la plateforme un espace réticulaire et hétérogène, qui donne forme à l'espace public de la consultation. C'est l'usage stratégique des liens hypertextes par des publics thématiques dans le cadre d'actions collectives qui constitue le ciment de cet espace.

Dans le cas de la CEC, le dispositif participatif est mis en ligne par les porteurs de projet qui publicisent son existence sur différents sites. Des réseaux thématiques, comme par exemple le réseau écologiste, ou celui de la légalisation du cannabis, vont trouver un intérêt particulier à participer à cette consultation : celui de faire connaître leurs actions et leurs positions sur une scène européenne. Sur un ou plusieurs sites intégrants ces réseaux, ils vont proposer des liens hypertextes qui conduisent vers la plateforme de la CEC (les grappins), à partir desquels les internautes vont pouvoir s'y rendre, notamment pour voter des propositions relatives aux sujets de mobilisation de ces réseaux thématiques. L'espace public en ligne de la consultation 
est alors constitué de l'ensemble des réseaux qui se raccordent au site de la CEC par des liens hypertextes. Le principe d'abordage met en avant la dimension indéterminable, imprévisible, de la constitution de cet espace puisque ce sont les participants eux-mêmes qui, successivement, en dessinent la forme.

Cette réflexion sur les formes prises par les espaces publics en ligne interroge directement les conceptions normatives de l'espace public européen portées par les dispositifs de la Commission. Dans le cas de projets comme la CEC, cet espace public est clairement considéré à travers un prisme procédural : il s'agit de réunir des citoyens, de les intégrer à une démarche participative structurée en étapes, et de canaliser leurs actions et leurs interactions. Ce prisme procédural qui place en son cœur la délibération doit beaucoup à l'approche habermassienne de l'espace public (Habermas, 1988 ; 1997) qui a inspiré de nombreuses expérimentations de démocratie participative. La Commission idéalise un citoyen " ordinaire », détaché de ses intérêts, qui agirait à titre individuel et se fonderait dans une entité collective construite en commun. Le dispositif doit donner corps à ce collectif et mettre en scène sa participation: c'est ainsi que la Commission entend construire un public européen, objectiver une opinion citoyenne, et performer un espace public transnational.

Les pratiques des internautes donnent à voir des modalités très différentes d'émergence des publics : ceux-ci se construisent autour d'intérêts partagés pour des thématiques spécifiques, par l'agrégation d'actions individuelles au sein d'un projet collectif. Les groupes se composent plus qu'ils ne se construisent (Callon, Lascoumes et Barthe, 2001): les individus sont engagés dans une dynamique où se définissent simultanément les contours du collectif et les entités qui le constituent. Ce mécanisme se produit à travers des modalités de mise en relation entre les individus, qui dans le cas étudié ici reposent sur le lien hypertexte comme mécanisme agrégateur. Le dispositif participatif est alors une scène publique qui autorise la manifestation des intérêts dans leur diversité et qui permet au collectif de se montrer à luimême. Ce faisant, il le fait exister. La plateforme participative performe le collectif, qui n'est plus « malgré lui », mais s'assume en agissant et en se voyant agir.

\section{Conclusion}

Si les actions collectives en ligne font preuve d'une grande hétérogénéité, il n'en demeure pas moins que l'usage stratégique du lien hypertexte en constitue une ressource structurante. Celui-ci intègre les répertoires d'action et intervient à la fois lors de la mobilisation d'un collectif, la conduite de l'action et la structuration d'un espace public autour d'une scène.

Il est un outil de mobilisation dans la mesure où, intégré à un message qui dresse le contexte de l'action collective, il constitue une offre pour des publics naviguant au sein de territoires thématiques et partageant un intérêt commun pour des sujets particuliers. Dans ce cadre, il est un canal de communication qui permet de faire circuler ces internautes depuis des espaces publics (des sites d'organisations), semi-publics (des groupes sur les réseaux sociaux), ou privés (des boîtes courriel sur lesquelles parviennent des listes de diffusion), vers la scène d'action.

Le lien hypertexte constitue également le mécanisme agrégateur qui structure l'action en intégrant des activités individuelles à un projet collectif. Cliquer le lien implique une interprétation par l'internaute de l'action qui est attendue de lui et traduit ainsi une forme d'engagement. L'usage du lien est donc éminemment stratégique : si les internautes qui 
diffusent des liens effectuent une proposition d'action, ceux qui les suivent valident leur participation à cette même action.

Le lien hypertexte va de ce fait structurer un espace public de la consultation, en articulant à la plateforme participative différents territoires thématiques. Cette connexion se fait via le principe d'abordage que nous avons décrit, dessinant ainsi un espace public opportuniste (il se construit à partir de l'opportunité offerte par la scène publique), temporaire (il ne dure que le temps de la consultation), hétérogène (il est constitué d'une multitude de territoires thématiques), et dont la forme est réticulaire (il est composé de réseaux de sites), indéterminable (il se constitue par abordages successifs et ne peut donc pas être planifié) et évolutive (il se transforme à chaque nouvel abordage).

Le lien hypertexte est donc constitutif des publics en ligne quand il structure les actions collectives dans lesquelles ces publics se composent. Les principales caractéristiques de ces actions en ligne touchent à la fois aux formes de l'engagement (l'agrégation par un clic), à la répartition égalitaire des ressources (militants "professionnels » et citoyens « ordinaires» utilisent des procédés similaires pour mobiliser «leurs» publics) et à la pertinence de la dimension thématique, plutôt que géopolitique, des territoires investis. Nous avons démontré ici que les spécificités du lien hypertexte n'y sont pas étrangères.

\section{Références bibliographiques}

AKRICH M. et MEADEL C. (2007), « De l'interaction à l'engagement : les collectifs électroniques, nouveaux militants dans le champ de la santé », Hermès, n47, p.145-154.

ALLARD L. et BLONDEAU O. (2007), Devenir Media : L'activisme sur Internet, entre défection et expérimentation, Paris, Editions Amsterdam.

BADOUARD R. (2013), "'Fabriquer" des publics européens en ligne", in ALDRIN P.et al, Les médiations de l'Europe politique, Presses Universitaires de Strasbourg, à paraître.

BADOUARD R. (2010), « Pathways and obstacles to e-Participation at the European level », Journal of eDemocracy and Open Government, 2(2), p.99-110.

BADOUARD R. et MONNOYER-SMITH L. (2013), "Hearing European Voices : The European Commission confronted with online activism », in Policy \& Internet special issue on Online Collective Action and Policy Change, à paraître.

BALME R., CHABANET D., WRIGHT V. (2002), L'action collective en Europe, Paris, Presses de Sciences Po.

BARABASI A.-L. (2002), Linked: The new science of networks, Perseus.

BARRY A. (2001), Political Machines. Governing a technological society, London, Athlone Press.

BENKLER Y. (2006), The Wealth of Networks: how social production transforms markets and freedom, Yale University Press. 
BENVEGNU N. et BRUGIDOU M. (2008), "Prendre la parole sur internet. Des dispositifs sociotechniques aux grammaires de la discussion", Réseaux, vol.4, n¹50, p.51-52.

BIMBER B., FLANAGIN A. J., et STOHL C. (2005), « Reconceptualizing collective action in the contemporary media environment », Communication Theory, $\mathrm{n}^{\circ} 15, \mathrm{p} .365-388$.

BOURE R., BOUSQUET F., 2011, « La construction polyphonique des pétitions en ligne. Le cas des appels contre le débat sur l'identité nationale ", Questions de communication, n²0, p.293-316.

BOURE R., BOUSQUET F., 2010, «Enjeux, jeux et usages d'une pétition politique. La pétition Vauzelle », Réseaux, n¹64, p.127-160.

BRUGIDOU M. (2008), L'opinion et ses publics : Une approche pragmatiste de l'opinion publique, Paris, Presses de Sciences Po.

CALLON M., LASCOUMES P., BARTHE Y. (2001), Agir dans un monde incertain. Essai sur la démocratie technique, Paris, Editions du Seuil.

CARDON D. (2010), La démocratie internet : Promesses et Limites, Paris, Ed. du Seuil.

CARDON D. (2008), "Le design de la visibilité. Un essai de cartographie du web 2.0", Réseaux, vol.6, ${ }^{\circ} 152$.

CARDON D., GRANJON F. (2010), Médiactivistes, Paris, Presses de Sciences Po.

CASTELLS Manuel, La société en réseau. L'ère de l'information, Paris : Fayard, 1998.

CASTELLS M. (2002), La galaxie internet, Paris, Fayard.

CEFAÏ D. (2007), Pourquoi se mobilise-t-on? Les théories de l'action collective, Paris, La Découverte/MAUSS.

CEFAÏ D., PASQUIER D. (dir.) (2003), Le sens du public : Publics politiques, publics médiatiques, Paris, Presses Universitaires de France.

CHAMPAGNE P. (1984), "La manifestation. La production de l'événement politique", Actes de la recherche en sciences sociales, vol. 52-53, p.19-41.

DE MAEYER J. (2011), "How to make sense of hyperlinks? An overview of link studies", OII-ICS Symposium, A decade in Internet time: symposium on the dynamics of internet and society, Oxford Internet Institute (Oxford, 21-24 September 2011).

FLANAGIN A. J., STOHL C., et BIMBER B. (2006), « Modeling the structure of collective action », Communication Monographs, vol. 73, p.29-54.

FLICHY P. (2011), Le sacre de l'amateur. Sociologie des passions ordinaires à l'ère numérique, Paris, Le Seuil.

FLICHY P. (2008), « Internet et le débat démocratique », Réseaux, vol. 4, n¹50, p. 159-185. 
FOUETILLOU G. (2008), « Le web et le traité constitutionnel européen : Ecologie d'une localité thématique compétitive », Réseaux, vol.1, n 147, p. 229-257.

GHITALLA F. (2008), « «La «Toile Européenne ». Parcours autour d'une cartographie thématique de documents web consacrés au thème de l'Europe et à ses acteurs sur le web francophone », Communication \& Langages, $\mathrm{n}^{\circ} 158, \mathrm{p} .61-75$.

GRANJON F. (2002a), "Les militants-internautes : passeurs, filtreurs et interprètes », Communication, $\mathrm{n}^{\circ} 22$, p.11-32.

GRANJON F. (2002b), «Les répertoires d'action télématiques du néo-militantisme », Le Mouvement Social, vol.3, n²00, p.11-32.

HABERMAS J. (1997), Droit et Démocratie. Entre faits et normes, Paris, Gallimard.

HABERMAS J. (1988), L'espace public, Paris, Payot.

IMIG S. et TARROW S. (2002), "La contestation politique dans l'Europe en formation », in BALME R., CHABANET D., WRIGHT V., L'action collective en Europe, Paris, Presses de Sciences Po, p.195-223.

ION J. (1997), La fin des militants?, Editions de l'atelier.

JACOMY M. et GHITALLA F. (2007), Méthodologies d'analyse de corpus en sciences humaines à l'aide du Navicrawler, Rapport final du programme TIC \& Migrations, Fondation Maison des Sciences de l'Homme, Août 2007.

JEANNERET Y. (2007) Y a-t-il (vraiment) des technologies de l'information ?, Lille, Presses Universitaires du Septentrion.

JENKINS H. (2006), Convergence Culture: Where Old and New Media Collide, New York University Press.

JOUËT J. (2011), « Des usages de la télématique aux Internet Studies », in DENOUËL J. et GRANJON F., Communiquer à l'ère numérique. Regards croisés sur la sociologie des usages, Presses des Mines.

LAURENT B. (2012), «Des assemblages incertains aux technologies de démocratie : deux modalités de l'expérience démocratique », Quaderni, nº79, p.81-98.

MARGETTS H., JOHN P. et ESCHER T. (2009), " Can the internet overcome the logic of collective action? An experimental approach to investigating the impact of social pressure on political participation», Paper to the Political Studies Association Annual Conference, University of Manchester, 7-9 April 2009.

MONNOYER-SMITH L. (2010), Communication et délibération. Enjeux technologiques et mutations citoyennes, Paris, Hermès Lavoisier, 2010.

NEVEU E. (1999), «Médias, mouvements sociaux, espaces publics », in Réseaux, vol. 17, 
$\mathrm{n}^{\circ} 98, \mathrm{p} .17-85$.

OLLITRAULT S. (1999), « De la caméra à la pétition-web : le répertoire médiatique des écologistes », Réseaux, vol.17, n 98, p.153-185.

QUERE L. (1992), «L'espace public : de la théorie politique à la métathéorie sociologique », Quaderni, ${ }^{\circ} 18$, pp. 75-92.

TILLY C. (1986), La France conteste de 1600 à nos jours, Paris, Editions Fayard.

WOJCIK S. (2011), « Participer... et après ? L’expérience des Consultations européennes des citoyens 2009 », Politique Européenne, n³4, 2011/2, p.135-166.

WRIGHT S. et STREET J. (2007), « Democracy, deliberation and design : the case of online discussion forums », New Media Society, n9, vol.5, p.849-870. 